4

\title{
Frailty, Swallowing and Dysphagia
}

\author{
David G. Smithard ${ }^{1,2} \cdot$ M. Shazra ${ }^{3} \cdot$ D. Hansjee ${ }^{1} \cdot$ I. Swaine ${ }^{2}$ \\ (C) Springer Science+Business Media, LLC, part of Springer Nature 2018
}

\begin{abstract}
Purpose of Review This paper is a brief overview of the relationship between frailty, swallowing and dysphagia. Its goal is to explore the interplay between age and sarcopenia in the development of dysphagia, which is known to be linked to aspiration pneumonia. It is postulated that there is growing justification for routine screening for dysphagia in older frail people, to enable rehabilitation of swallowing through exercise and nutritional intervention, after a hospital stay.

Recent Findings The global population is ageing, with a particular increase in the very old and frail. Frail people have a limited functional and physiological reserve and often have sarcopenia. Any subsequent insult (trauma, illness, medication change) frequently results in decompensation and the need for a hospital stay. Often, in these patients, there are changes in the biomechanics of swallowing that can cause impairment and dysphagia. But, many patients adapt the way they eat with subtle compensatory techniques, to bypass this difficulty. It is possible that many more people, than is currently evident, have undiagnosed dysphagia. Pneumonia and respiratory disease are common reasons for hospital admission in the frail elderly population. Dysphagia with aspiration is an important aetiological factor in pneumonia, which is a serious health concern with increasing age. Dysphagia may simply be a consequence of physiological decompensation, related to age, frailty and sarcopenia. Dysphagia is not systematically screened for and may not be identified in many older frail people who have adapted their swallowing, to accommodate their dysphagia. This may be a significant factor in pneumonia-related hospital admissions. Swallow rehabilitation, after such admission to hospital, is also rarely offered in the acute medical setting. This needs to change to reduce recurrent admission, morbidity and mortality.

Summary The population is ageing. Sarcopenia, frailty and dysphagia are common with increasing age. Pneumonia is a common admission to hospital and often, aspiration secondary to dysphagia is a common cause. Proactive identification and intervention has the potential to reduce morbidity, hospital admission, length of hospital stay and mortality.
\end{abstract}

Keywords Frailty $\cdot$ Dysphagia $\cdot$ Exercise $\cdot$ Nutrition $\cdot$ Inflammation

\section{Introduction}

The global population is expanding in an asymmetric way, with an increase in older and in particular very old people. This is driven, in part by the improvements in public health and acute medical care. As the population ages, so the number

This article is part of the Topical Collection on Swallowing Disorders

David G. Smithard

david.smithard@nhs.net

1 Queen Elizabeth Hospital, Woolwich, London, UK

2 University of Greenwich, London, UK

3 Princess Royal University Hospital, Farnborough, Kent, Orpington, UK of people with long-term conditions increases. This ultimately results in more people becoming frail, such that $50 \%$ of those over the age of 80 years are categorised as frail [1]. Frailty is described as a general decline in physiological function associated with loss of muscle bulk, weakness, fatigue and slowness. Sarcopenia frequently accompanies frailty and is also associated with muscle weakness, loss of skeletal muscle mass and poor quality of muscle fibres.

Frailty is a complex syndrome associated with a progressive decline in physical, mental and social functions. There is increased vulnerability to deterioration and reduced potential for recovery $[1,2]$ associated with sarcopenia, undernutrition, slowness and disability. Frail older people have little or no physiological reserve, such that whenever they are exposed to an insult (medication change, constipation, trauma and acute illness) physiological and functional decompensation results. The insult may be minor, but due to the lack of reserve, 
it will result in reduced mobility, confusion/delirium and frequently, dysphagia. Frail older people also have multiple comorbidities, increased mortality [3] and have a limited physiological reserve. Any illness will result in decompensation [4, 5], which may include the development of difficulties in swallowing (dysphagia).

The International Classification of Functioning, Disability and Health [6] classifies normal swallowing as 'functions of clearing the food and drink through the oral cavity, pharynx and oesophagus into the stomach at an appropriate rate and speed'. As the bolus (food or liquid) passes through the mouth and pharynx, it is subject to multiple pressures, which are dependent on the function of the tongue and upperoesophageal sphincter (UES), pharynx and influenced by the inherent properties of the bolus.

\section{Frailty-Related Dysphagia}

Frail older people have muscle loss and sarcopenia; the loss of appendicular skeletal muscle is easy to recognise. The suprahyoid muscles, which are involved in swallowing (assisting in tongue movement, laryngeal elevation and anterior movement and UES opening [7]), are also skeletal muscles and will be affected by sarcopenia. This is often forgotten, or medical staff is not aware of the fact. The consequences of this include reduced elevation and anterior movement of the larynx and reduced opening aperture of the UES, which results in a potentially less safe swallow than in younger adults [8]. A smaller UES aperture and reduced pharyngeal stripping wave pressures result in food residue remaining in the pharynx after the swallow, increasing the risk of post-swallow aspiration $[4,7,9,10]$.

Frail older adults have complex medical needs. Frequently people have multiple, co-existing, long-term medical conditions that will impinge on the eating, drinking and swallowing. For example, rheumatoid arthritis can affect the arytenoid joints, thereby affecting closing of the vocal cords [11]. Swallowing requires a short period of breath holding; in the context of cardio-respiratory disease can lead to hypoxia and secondary dysphagia; head and neck cancer; stroke, vascular or Alzheimer's dementia can all lead to changes in the oral or pharyngeal phases of the swallow. A dry mouth secondary to medication, mouth breathing, radiotherapy or auto immune disease can all affect the consistency of food eaten.

The aetiology of sarcopenia and frailty is complex often involving neurohumoral pathways (suggesting inflammatory pathway involvement), poor diet and lack of exercise. Therefore, it should be feasible to break the downward spiral/cycle which will ultimately result in death, through lifestyle change. This could help to avoid the need for a hospital stay every time a frail person becomes unwell. When this occurs, the functional ability of the older frail person declines and recovery may take many months. Often, the end result, physically and cognitively, may be a person who is more dependent than previously.

To reduce and/or ameliorate the presence of dysphagia and its complications in this older cohort, strategies to combat frailty need to be developed.

\section{Exercise}

There is increasing evidence to suggest that exercise can counteract some of the effects of frailty on physical function. Many expert groups are recommending exercise for frail older people. Indeed, a recent review suggested that exercise consistently combats the deleterious effects of sarcopenia. Most reviews have also suggested that resistance exercise, for musclestrengthening, is more efficacious in this regard [12].

Muscle mass and strength can be maintained and increased by aerobic and resistance exercise (including isometric exercise). For example, taking up dancing can improve balance and coordination, singing can help respiratory muscle strength and walking can improve aerobic capacity and leg muscle strength. Exercise, commenced in or before middle age, has been shown to protect against circulatory disease (stroke and heart). In the case of appendicular sarcopenia, other types of exercise may prevent or improve muscle bulk and strength [1, 13-15].

It is therefore plausible that a 'general' exercise programme could help to counteract frailty-related dysphagia. Improvement in cardiorespiratory fitness and general muscle strength could have an indirect effect on the aerobic function and strength of the swallowing musculature. However, more-targeted, specific exercise programmes have been developed to improve swallowing. These exercise programmes target the swallowing muscles (e.g. hyoid muscles) usually by requiring people to perform chin tuck movements against some type of resistance. Because this type of exercise usually involves a 'static phase' (where the movement is held stationary against the resistance, for example, for $30 \mathrm{~s}$, or 1-2 min), these exercises are sometimes described as 'isometric resistance'.

Indeed, for dysphagia, Shaker and colleagues developed a system of exercises to train the hyoid group of muscles and neck muscles [16]. Shaker exercise resulted in an increase in thyrohyoid shortening after 6 weeks compared to tongue exercises and swallowing manoeuvres [17]; increases in contraction pressures in the pharynx, increased opening of the UES, [18, 19] occurs. However, the studies are small, and more data is required to determine how chin tuck affects the physiology of swallowing.

Therefore, it may be entirely possible to significantly affect dysphagia in the frail older person through general and targeted exercise programmes. The mechanism whereby this occurs needs to be further elucidated, but would probably involve improved oxygen supply, muscle strength, endurance, coordination and neurohumoral control of movement. The most appropriate way to achieve these effects, in relation to frail older 
154 people with dysphagia, is not yet known. This is largely because 155 so few studies have explored this idea in this group of people.

\section{Nutrition}

Malnutrition is associated with increased infection risk and poor wound healing. Malnutrition and dehydration will result in muscle weakness and hypotension and an increased risk of falls. Falls in frail older people, place them at risk of subdural haematomas and fractured neck of femurs, both of which carry a significant morbidity and mortality.

Improvement in nutrition and calorie intake will help in the anabolic effect provided by exercise. Older adults are inefficient in the utilisation of amino-acids from protein and are also inefficient in energy usage and hence older people require a balanced diet to maintain weight, muscle mass, strength, and to improve immunity.

\section{Inflammation}

More recently there has been interest shown in the interruption of the inflammatory process (raised CRP, interleukins and cytokines) present in sarcopenia and frailty, to arrest the process. Some work has shown a benefit in the use of antiinflammatory agents (Ibuprofen). However, there is limited evidence of clinical benefit [20].

\section{Dysphagia}

Various studies have noted a frequency of up to $30 \%$ of older people living at home may have dysphagia [21] with up to $28 \%$ of older people noted to be aspirating on instrumentation $[22,23]$. The frequency of swallowing problems in older people is uncertain as many older people do not report problems [4] or have accepted them as a fact of life [24] and have often subtly and unknowingly compensated for motor changes [4].

Many older frail people admitted to hospital (55\%) will have difficulties with swallowing (dysphagia) [25]. Up to $90 \%$ of those admitted with a diagnosis of pneumonia, may have inhaled saliva or food into the lungs (aspiration). Periodontal disease is not uncommon, with rotten teeth and gum infection, which will increase the risk of pneumonia secondary to the inhalation of infected saliva. Recurrent infection will result in a decreased lung function and also a worsening of functional state following each infection.

Swallowing problems/dysphagia, when present in older people, is poorly managed in many institutionalised settings including the acute hospital. In many European countries, the presence of dysphagia (or difficulties with eating and drinking) in frail people is not routinely sought. There is no requirement to systematically screen frail patients for the presence of dysphagia as there is in stroke patients [26, 27]. This is a missed opportunity, by policy makers, to potentially improve outcome and reduce hospital stay and re-admissions.

\section{Routine Screening for Dysphagia in Frail Older People}

If frail patients are to be screened for dysphagia, on admission to hospital, which swallow screen should be used? There are many swallowing screens available to clinicians (Medical, SLP, AHP, Nursing), many which are validated, but many staff continue to use locally developed, non-validated tools [28]. These tools have been validated in the stroke population and recently, evidence has been published supporting the use of TOR-BSST in care homes [29].

The swallow screens that are available are generally very similar. They all have the same aim, of a clinical/anatomical assessment, a trial of small volumes of water followed by a larger volume. Some are simple, with others being more complex. Recent work that we have conducted has shown that the most characteristic identifiers are coughing and choking when swallowing, taking longer to drink, a change in diet and a change in voice quality.

\section{Proactive Intervention}

Implementing a policy of screening will not improve patient outcomes unless there is a positive proactive intervention. Standard hospital rehabilitation, for dysphagia, includes postural manoeuvres (e.g. Chin-Tuck). It has been accepted that the Chin Tuck manoeuvre pulls the larynx up and forwards and at the same time opens the UES [17, 30]. Welch et al. [30] also claimed that there was posterior movement of the tongue base towards the posterior pharyngeal wall. Momasaki et al. [31] using a large Japanese database demonstrated that those patients with dysphagia, who were offered appropriate rehabilitation, were more likely to have a total oral intake compared to those not offered oral-pharyngeal rehabilitation (OR $1.2 P<0.001)$. Pogus-Pulia et al. [32] demonstrated, using isometric progressive resistance oropharyngeal therapy [33], improved FOIS (Functional Oral Intake Score- a measure of food intake [34] effect estimate $=0.4, p<0.02$ ), reduced incidence of pneumonia and reduced number of hospital admissions. Their cohort was mixed in aetiology of dysphagia.

\section{Mouth Care}

Dentition and mouth care are important factors to consider. Lack of teeth will make chewing more difficult, resulting in

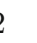

3

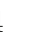

5

7

8

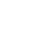

2

4

6

7

19


modifications to the food choices eaten. This may mean blending what is usually eaten, or switching to softer and possibly nutritionally poorer foods. For those who are edentulous, dentures are often provided (where available), but in many cases are not worn due to discomfort, or are not kept clean. Unhygienic dentures could result in candidiasis and in dysphagia due to pain.

\section{End of Life}

Frailty carries a significant mortality, with those with extreme frailty [35] having a short life span. The management of dysphagia in this group will move fairly rapidly from active management to a palliative care approach. There will be a move from ensuring adequate calories, to that of offering food/liquid for comfort as and when the person is able and in volumes that are safe. There is little consensus on terminology with respect to this approach and terms include 'finger feeding' and 'risk feeding'. How this is approached will vary depending on societal, cultural, religious sensibilities and expectations.

\section{Conclusions}

Worldwide, by $2050,34 \%$ of the world's 9.7 billion population there will be $>60$ years, with 125 million $>80$ years [36]. Fifty percent of older adults $>80$ years older adults will be frail and prone to deteriorating health and dependence. It is possible to slow down the march to frailty and dependence, particularly in the pre-frail phase, by implementing a programme of exercise and good nutrition. The presence of dysphagia is likely to be high in this population; routine screening for problems, eating, drinking and swallowing should be undertaken in primary care and when an older frail person is admitted to hospital.

\section{Compliance with Ethical Standards}

Conflict of Interest The authors declare that they have no competing interests.

Human and Animal Rights and Informed Consent This article does not contain any studies with human or animal subjects performed by any of the authors.

\section{References}

1. Phu S, Boersma D, Duque G. Densitometry: assessment and management of musculoskeletal health. J Clin. 2015;18:1-5.

2. Fried LP, Tangen CM, Walston J, Newman AB, Hirsch C, Gottdiener J, et al. Frailty in older adults: evidence for a phenotype. J Gerontol. 2001;56:M146-57.
3. Stanaway FF, Gnjidic D, Blyth FM, Le Couteur DG, Naganathan V, Waite L, et al. How fast does the grim reaper walk? Receiver operating charcteristics curve analysis in healthy men aged 70 and over. BMJ. 2011;343:d7679.

4. Omari TI, Kritas S, Cock C, Besanko L, Burgstad C, Thompson A, et al. Swallowing dysfunction in healthy older people using pharyngeal pressure-flow analysis. Neurogastroenterol Motil. 2014;1: 59-68.

5. Nicosia MA, Hind JA, Roecker EB, Carnes M, Doyle J, Dengel GA, et al. Age effects on the temporal evolution of isometric and swallowing pressure. J Gerontol: Medi Sci. 2000;55A:M634-40.

6. The International Classification of Functioning, Disability and Health (ICF). 2001 WHO Geneva http://apps.who.int/iris/ bitstream/10665/42407/7/9241545429_tha\%2Beng.pdf

7. Curtis JA, Laus J, Yung KC, Courey MS. Static endoscopic evaluation of swallowing: transoral endoscopy during clinical swallow evaluations. Laryngoscope. 2016;126:2291-4.

8. Dejaeger M, Liesenborghs C and Dejaeger E Presbyphagia. In Seminars in Dysphagia. InTech; 2015.

9. Curtis J, Langenstein J, Scheider S. Superior and anterior displacement during swallowing in non-dysphagic individuals. Dysphagia. 2018; https://doi.org/10.1007/s00455-018-9878-7.0123456789.

10. Sze WP, Yoon WL, Escoffier N. Liow evaluating the training effects of two swallowing rehabilitation therapies using surface electromyography — chin tuck against resistance (CTAR) exercise and the shaker exercise. Dysphagia. 2016;31:195-205.

11. Gleason JB, Hadeh A. Vocal hoarseness in rheumatoid arthritis: early recognition is critical. J Clin Diagn Res. 2017;11(4):OJ03.

12. Theou O, Stathokostas L, Roland KP, Jakobi JM, Patterson C, Vandervoort AA, et al. The effectiveness of exercise interventions for the management of frailty: a systematic review. J Aging Res. 2011, Article ID 569194, 19 pages;2011:1.

13. Otsuka R, Matsui C, Tange C, Nishita Tomida M, Fujiko A, Shimokata $\mathrm{H}$, et al. What is the best adjustment of appendicular mass for predicting mortality or disability among Japanese community dwellers? BMC Geriatr. 2018;18:8. https://doi.org/10.1186/ s12877-017-0699-6.

14. Komi PV, Viitasalo JT, Rauramaa R, et al. Europ. J Appl Physiol. 1978;40:45.

15. Anwer S, Alghadir A. Effect of isometric quadriceps exercise on muscle strength, pain, and function in patients with knee osteoarthritis: a randomized controlled study. J Phys Ther Sci. 2014;26(5): $745-8$.

16. Shaker R, Eastreling C, Kern M, Nitschke T, Massey B, Daniels SK, et al. Rehabilittaion of swallowing by exercises in tube-fed patients with pharyngeal dysphagia secondary to abnormal UES opening. Gastroenterology. 2002;122:131401321.

17. Mapani R, Antonik S, Massey B, Kern M, Logemann J, Pauloski B, et al. Augmentation of deglutitive thyrohyoid muscle shortening by the Saker exercise. Dysphagia. 2009;24:26-31.

18. Balaou M, McCullough GH, Aduli F, Brown D, Stack BC, Snoddy $\mathrm{P}$, et al. Manometric measure of head rotation and chin tuck in healthy participants. Dysphagia. 2014;29:25-32.

19. McCullough GH, Kamarunas E, Mann GC, Schmidley JW, Robbins JA, Crary MA. Effects of Mendelsohn maneuver on measures of swallow duration post stroke. Top Stroke Rehabil. 2012;19:234-43.

20. Li H, Manwani B, Leng SX. Frailty, inflammation, and immunity. Ageing Dis. 2011;2:466-73.

21. Kertscher B, Speyer R, Fong E, Georgiou AM, Smith M. Prevalence of oropharyngeal dysphagia in the Netherlands: a telephone survey. Dysphagia. 2015;30:114-20.

22. Butler SG, Stuart A, Leng X, Rees C, Williamson J, Kritchevsky SB. Factors influencing aspiration dur- ing swallowing in healthy older adults. Laryngoscope. 2010;120:2147-52. 
31. Momosaki R, Yasunaga H, Matsui H, Horiguchi H, Fushimi K, Abo M. Effect of dysphagia rehabilitation on oral intake in elderly patients with aspiration pneumonia. Geriatr Gerontol Int b2014.

32. Rogu-Pulia N, Rusche N, Hind JA, Zielinski J, Gangnon R, Safdar $\mathrm{N}$, et al. Effects of device-facilitated isometric progressive resistance oropharyngeal therapy on swallowing and health related outcomes in older adults with dysphagia. JAGS. 2016;

33. Robbins J, Kays SA, Gangnon RE, Hind JA, Hewitt AL, Gentry LR, et al. The effects of lingual exercise in stroke patients with dysphagia. Arch Phys Med Rehabil. 2007;88(2):150-8.

34. Crary MA, Carnaby Mann GD, Groher ME. Initial psychometric assessment of a functional oral intake scale for dysphagia in stroke patients. Arch Phys Med Rehabil. 2005;586:1516-20.

35. Mitnitski A, Graham J, Mogilner A, Rockwood K. Frailty, fitness and late-life mortality in relation to chronological and biological age. BMC Geriatr. 2002;2:1-8.

36. United Nations, Department of Economic and Social Affairs, Population Division (2015). World Population Ageing 2015 (ST/ ESA/SERA/390).

37. Artaza-Artabe I, Saez-Lopez P, Sanchez-Hernandez N, FernandezGutierrez N, Malafarina V. The relationship between nutrition and frailty: effects of protein intake, nutritional supplementation, vitamin D and exercise on muscle metabolism in the elderly: a systematic review. Maturitas. 2016;93:89-99. Acad Nurs. 2015;45:1-13.

30. Welch MV, Logemann JA, Rademaker AW, Kahrilas PJ. Changes in pharyngeal dimensions effected by chin tuck. Arch Phys Med Rehabil. 1993;74:78-81. 